\title{
Endometrial thickness cut-off value by transvaginal ultrasonography for screening of endometrial pathology in premenopausal and postmenopausal women
}

\author{
Yu Ran Park', Shin Wha Lee'2, Yeongsin Kim', In Young Bae', Hong-Kyu Kim', Jaewon Choe', Yong-Man Kim² \\ ${ }^{1}$ Health Screening and Promotion Center, ${ }^{2}$ Department of Obstetrics and Gynecology, Asan Medical Center, University of Ulsan College of Medicine, \\ Seoul, Korea
}

\section{Objective}

To assess the clinical usefulness and diagnostic accuracy of ultrasonographic measurement of endometrial thickness (ET) in women with endometrial hyperplasia or cancer $(\mathrm{EH}+)$.

Methods

This retrospective cohort study included 29,995 consecutive women who underwent transvaginal ultrasonography (TVS) for an incidental finding of a thickened endometrium at the health screening and promotion center at Asan Medical Center between 2006 and 2010. Among 959 patients with endometrial abnormalities, 92 patients were included in this study. A total of 867 patients were excluded: 416 were lost to follow-up; 263 did not undergo endometrial biopsy; 155 had endometrial polyps; 17 had submucosal myomas; and 16 had insufficient tissue samples. Endometrial histology was the reference standard for calculating accuracy.

Results

Of the 92 patients, $78(84.8 \%)$ had normal pathology, while $14(15.2 \%)$ had endometrial pathology (EH+), including 5 patients (35.7\%) with simple hyperplasia without atypia, $3(21.4 \%)$ with complex hyperplasia, and $6(42.9 \%)$ with endometrial carcinoma, all stage la. The area under the receiver-operating characteristic curve was $0.75(95 \%$ confidence interval [Cl], 0.593-0.906). The cut-off value for ET was $8 \mathrm{~mm}$, indicating that TVS ET had a fair accuracy in diagnosing carcinoma, had a sensitivity of $100 \%(95 \% \mathrm{Cl}, 62.9-100.0 \%)$ and a specificity of $24.3 \%(95 \% \mathrm{Cl}, 15.2-$ $36.3 \%)$.

\section{Conclusion}

TVS is useful for detecting EH+, with a cut-off value for ET of $8 \mathrm{~mm}$ having a high sensitivity for detecting endometrial pathologies and the ability to identify women highly unlikely to have $\mathrm{EH}+$, thereby avoiding more invasive endometrial biopsy.

Keywords: Diagnosis; Endometrium; Endometrial hyperplasia; Endometrial neoplasms

\section{Introduction}

Endometrial cancer is the most common gynecologic malignancy. The worldwide incidence of endometrial cancer has risen over the last 20 years [1]. Increases in the rates of obesity and decreases in the rates of fertility suggest that the incidence of endometrial cancer will continue to increase in postmenopausal women, becoming a substantial public health problem worldwide $[2,3]$. This rise in its incidence has implications for both primary prevention and screening.

Although vaginal bleeding is the most common symptom
Received: 2018.10.19. Revised: 2019.04.12. Accepted: 2019.05.28. Corresponding author: Shin Wha Lee

Department of Obstetrics and Gynecology, Asan Medical Center, University of Ulsan College of Medicine, 88 Olympic-ro 43-gil,

Songpa-gu, Seoul 05505, Korea

E-mail: swhlee@amc.seoul.kr

https://orcid.org/0000-0002-5088-1905

Articles published in Obstet Gynecol Sci are open-access, distributed under the terms of the Creative Commons Attribution Non-Commercial License (http://creativecommons. org/licenses/by-nc/3.0/) which permits unrestricted non-commercial use, distribution, and reproduction in any medium, provided the original work is properly cited.

Copyright $\odot 2019$ Korean Society of Obstetrics and Gynecology 


\section{Obstetrics \& Gynecology Science}

Vol. 62, No. 6, 2019

in patients with endometrial cancer, up to $20 \%$ of patients who are diagnosed with endometrial cancer are asymptomatic at the time of diagnosis [4]. Moreover, it is often difficult to define abnormal uterine bleeding in perimenopausal women, who usually experience irregular menstruation as their ovarian function declines; in addition, clinicians have differing assessments regarding the exact mechanism constituting the perimenopausal state [5-7].

Many studies have validated the use of transvaginal ultrasonography (TVS) as the initial screening method for endometrial cancer [8-11]. Although an endometrial thickness (ET) of $\geq 5 \mathrm{~mm}$ is regarded as the cut-off value for postmenopausal women who present with vaginal bleeding, it warrants further investigation [12]. Furthermore, there is no established consensus on the ET threshold that distinguishes normal from malignant pathology in postmenopausal women without bleeding. Because the factors associated with a thickened endometrium in these women remain undetermined, the clinical management of women with an incidentally detected thickened endometrium has not been standardized or established. TVS is usually requested by general practitioners as a part of the general investigation of women complaining of various symptoms involving the abdomino-pelvic region, and the cut-off value for ET is unclear under various clinical conditions. Therefore, women with vaginal bleeding or spotting may undergo repeated TVS and unnecessary invasive diagnostic tests.

This study was designed to evaluate the diagnostic value of ET as a predictor of endometrial carcinoma in women undergoing health examination. The optimal cut-off values distinguishing between women with and without intrauterine pathologies were determined. The knowledge of these cutoff values may avoid the need to perform more invasive and unnecessary diagnostic tests, such as endometrial biopsy.

\section{Materials and methods}

\section{Patients}

Data from 29,995 consecutive patients who underwent pelvic ultrasonography between January 2006 and December 2010 at the health screening and promotion center in Asan Medical Center, Seoul, South Korea were retrieved retrospectively. Of these, 959 (3.2\%) were found to have an endometrial abnormality on TVS and were recommended to undergo follow-up gynecologic examinations, including endometrial biopsy, such as dilated curettage and biopsy or hysteroscopic endometrial biopsy. Of the 959 patients, 867 (90.4\%) were excluded from the analysis, including 416 who were lost to follow-up, 263 who did not undergo endometrial biopsy, 155 with endometrial polyps, 17 with submucosal myomas, and 16 who lacked sufficient tissue samples for obtaining adequate results. Thus, this study included 92 patients who underwent endometrial biopsy. Fig. 1 shows the patient flow diagram.

\section{Clinical assessment and measurements of endometrial abnormality}

The medical records of all patients, including their detailed medical history and results of physical examination, were reviewed. The data collected included patient age, height, body weight, body mass index, systolic blood pressure (BP), diastolic BP, and waist circumference. Medical history included information on prior use of hormones, herbs, or tamoxifen; history of breast cancer and gynecologic cancer; menopausal status; and occurrence of diabetes, hypertension, hyperlipidemia, and vaginal bleeding. The incidence of endometrial hyperplasia or cancer $(\mathrm{EH}+)$ was determined by reviewing the electronic medical records of each patient from her first visit to December 31, 2016.

All TVS examinations were performed using the SSD 5000SV (ALOKA, Tokyo, Japan) ultrasound machine, equipped with a 3-9-MHz transvaginal transducer. The ET was measured at the mid-sagittal plane from the outer borders of the anterior and posterior endometria at the thickest part. All scans were performed by 2 certified gynecologists who had completed the obstetrics and gynecologic departmental training requirements and assessments.

Endometrial pathology on TVS was defined as the appearance of a diffusely thickened, homogeneously echogenic endometrium; focal endometrial thickening; or cystic changes presenting as small anechoic foci. The cut-off value for ET was $\geq 5 \mathrm{~mm}$ in postmenopausal women [12]. The cut-off values for premenopausal women were $\geq 8 \mathrm{~mm}$ in the proliferative phase and $\geq 16 \mathrm{~mm}$ in the secretory phase. If the patients had their last menstruation within 12 months but forgot their exact last menstrual period (LMP) or could not expect their next menstruation date, we set the cut-off value at $\geq 8 \mathrm{~mm}$. 


\section{Obstetrics \& Gynecology Science}

Yu Ran Park, et al. Endometrial thickness by sonography

\section{Diagnosis of endometrial pathology}

All histopathological examinations were performed by pathologists not involved in the study and not blinded to the patients' pelvic ultrasonographic findings. The clinical information of each patient was recorded and made available within the hospital's centralized electronic medical records system at the time of patient assessment. Reports of endometrial curettage and hysterectomy were reviewed for all patients, with these details constituting the reference standard for endometrial histology.

\section{Statistical analysis}

Baseline variables were presented as numbers (with percentages) and means (with standard deviations [SDs]). Continuous variables were compared using Student's $t$-test and categorical variables using the $\chi^{2}$ or Fisher's exact test. All statistical calculations were 2-sided, with a $P$-value of $<0.05$ considered statistically significant. Receiver-operating characteristic (ROC) curves were calculated to assess whether ET measurement on TVS was diagnostic of endometrial pathology. All statistical analyses were performed using the SPSS software package, version 21 (SPSS Inc., Chicago, IL, USA).

\section{Results}

\section{Patients and pathological characteristics}

The 92 included patients had a median follow-up duration of 2.981 years (interquartile range, $0.463-6.444$ years; range, 5 days to 10.729 years). The mean frequency of repeated TVS during follow-up was 4.912 (median, 4.000).

Table 1 shows the baseline characteristics of the 92 study patients. Of these women, $14(15.2 \%)$ were diagnosed with endometrial pathology $(\mathrm{EH}+)$, and 78 (84.8\%) were assessed to have normal pathology based on the results of the final pathological analysis of the endometrial biopsy samples. The mean ages of the patients in these 2 groups were 47.8 (SD, $6.8)$ and 50.4 (SD, 7.9) years, respectively. Three patients $(21.4 \%)$ in the $\mathrm{EH}+$ group and $41(52.6 \%)$ in the normal group were menopausal $(P<0.05)$.

Of the 14 patients in the EH+ group, 5 (35.7\%) had simple hyperplasia without atypia; 3 (21.4\%) had complex hyperplasia; and 6 (42.9\%) had endometrial carcinoma, including 4 with endometrioid adenocarcinoma, 1 with clear cell carcinoma, and 1 with mixed cell carcinoma (uterine papillary serous carcinoma, endometrioid adenocarcinoma, and clear cell carcinoma). Only 5 (35.7\%) patients in the $\mathrm{EH}+$ group

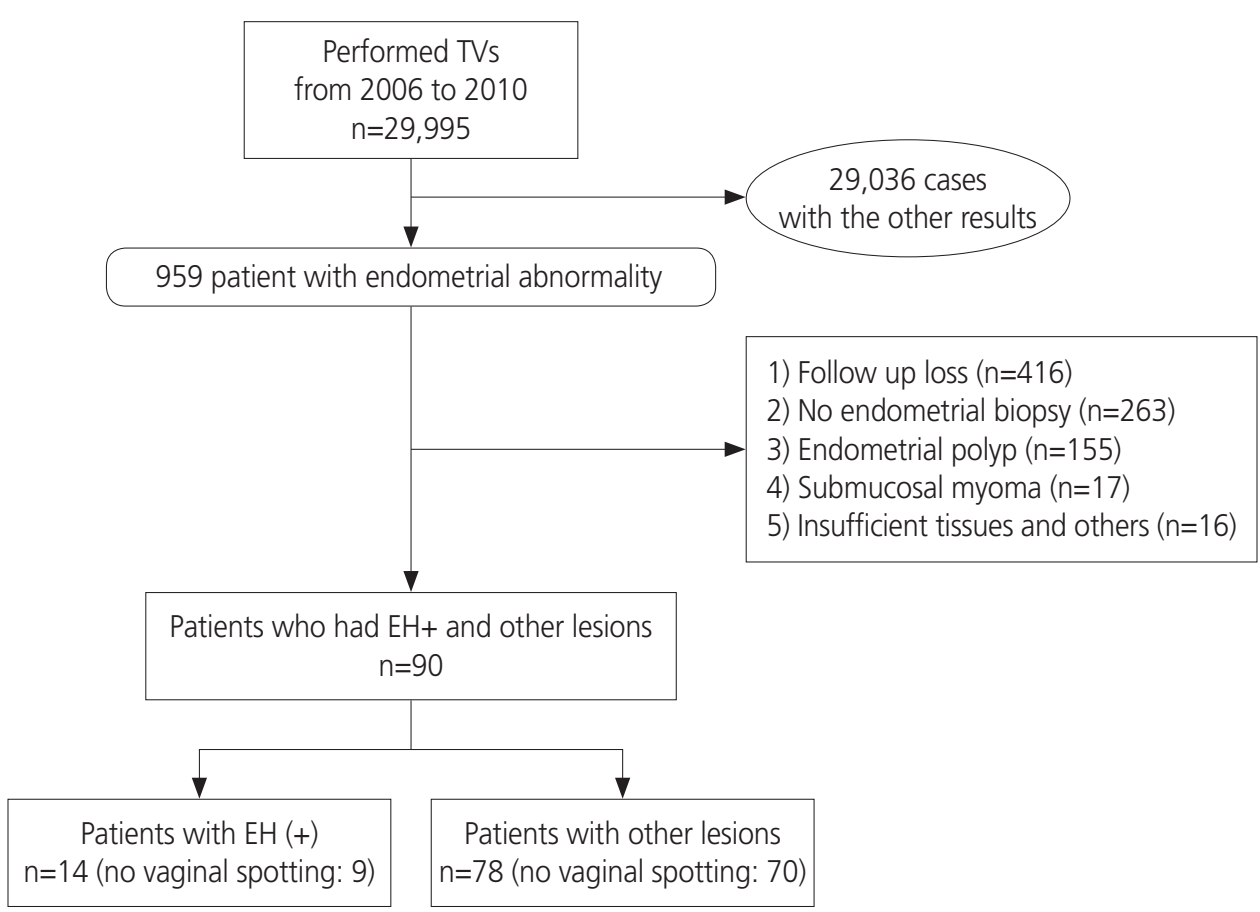

Fig. 1. Flow diagram of cohort selection for assessing the diagnostic accuracy of endometrial thickness. EH+, endometrial hyperplasia or cancer. 


\title{
Obstetrics \& Gynecology Science
}

\author{
Vol. 62, No. 6, 2019
}

had symptoms of vaginal bleeding or spotting. Three patients were in the postmenopausal state; 2 had simple hyperplasia; and 1 had endometrial cancer. All patients with endometrial cancer were treated surgically by hysterectomy and bilateral salpingo-oophorectomy, optional pelvic lymph node dissection, and para-aortic lymph node sampling. Five of the 6 patients with endometrial cancer had stage la tumors and underwent no further treatment; the patient with clear cell carcinoma received 6 cycles of adjuvant chemotherapy, consisting of a combination of doxorubicin and cisplatin. The clinicopathological characteristics of the patients in the $\mathrm{EH}+$ group are presented in Table 2.

\section{Diagnostic value of endometrial thickness and receiver-operating characteristic curve}

Fig. 2 shows the ROC curve for the estimated diagnostic performance of the TVS ET measurements in detecting endometrial pathology. The area under the ROC curve was 0.749 (95\% confidence interval $[\mathrm{Cl}], 0.593-0.906 ; P<0.001$ ).

Table 3 shows the efficiency of the different cut-off values on TVS in detecting endometrial pathology. The cutoff values of 6,7 , and $8 \mathrm{~mm}$ had sensitivities of $100 \%$ (95\% Cl, 62.9-100.0\%) and specificities of $2.9 \%(95 \% \mathrm{Cl}$, $0.1-10.9 \%), 11.4 \%(95 \% \mathrm{Cl}, 0.5-21.8 \%)$, and $24.3 \%$ (95\% $\mathrm{Cl}, 15.2-36.3 \%)$, respectively, in the detection of endometrial cancer. The false-positive rates of these cut-off values were $88.3 \%(95 \% \mathrm{Cl}, 78.5-94.2 \%), 87.3 \%(95 \% \mathrm{Cl}, 7.68-$ $93.7 \%)$, and $85.5 \%(95 \% \mathrm{Cl}, 73.7-92.7 \%)$, respectively.

\section{Discussion}

TVS is used as the initial screening method for endometrial pathology; it has replaced dilatation and curettage as the first-line investigation method for women with incidental vaginal bleeding and spotting. TVS has been the technique of choice for endometrial evaluation of postmenopausal bleeding over the last 2 decades because of its high accuracy and non-invasive nature [13]. However, in the general population, there is currently no established screening method for endometrial cancers and pathologies. Women at an average or increased risk because of a history of unopposed estrogen therapy, tamoxifen therapy, late menopause, nulliparity, infertility, obesity, diabetes, and hypertension should be informed of the risks and symptoms of endometrial pathologies and encouraged to report these symptoms to their physicians [14]. The present guidelines recommend that only women with Lynch syndrome, which is a genetic disorder with a lifetime endometrial cancer risk of $40-60 \%$, must undergo screening for endometrial cancer [15].

Furthermore, there is no consensus on the cut-off value for ET for detecting the presence of any abnormality. No screening guidelines for endometrial pathology have also been formulated for pre- and perimenopausal women who experience irregular menstruation or minimal vaginal spotting. Rather, they undergo TVS only after massive, repetitive vaginal bleeding or frequent irregular menstruation. Because endometrial cancer can occur in the absence of bleeding,

Table 1. Baseline characteristics in the endometrial hyperplasia or cancer $(\mathrm{EH}+)$ and normal groups

\begin{tabular}{lccc}
\hline Characteristics & EH $(+\mathbf{)}(\mathbf{n}=\mathbf{1 4})$ & Normal $(\mathbf{n = 7 8 )}$ & $\boldsymbol{P}$-value \\
\hline Age $($ yr) & $47.8 \pm 6.8$ & $50.4 \pm 7.9$ & 0.184 \\
Menopause & $3(21.4)$ & $41(52.6)$ & 0.032 \\
Hypertension & $5(35.7)$ & $13(16.7)$ & 0.098 \\
Diabetes mellitus & $0(0.0)$ & $4(5.1)$ & 0.386 \\
Previous hormone replacement therapy & $0(0.0)$ & $5(6.4)$ & 0.330 \\
Height $(\mathrm{cm})$ & $159.6 \pm 5.7$ & $158.7 \pm 4.6$ & 0.543 \\
Body weight $(\mathrm{kg})$ & $58.5 \pm 11.5$ & $58.6 \pm 8.6$ & 0.952 \\
Body mass index $\left(\mathrm{kg} / \mathrm{m}^{2}\right)$ & $23.0 \pm 4.2$ & $23.3 \pm 3.2$ & 0.754 \\
Systolic BP $(\mathrm{mmHg})$ & $115.6 \pm 24.6$ & $112.9 \pm 14.0$ & 0.700 \\
Diastolic BP $(\mathrm{mmHg})$ & $67.8 \pm 12.6$ & $69.6 \pm 10.6$ & 0.577 \\
Waist circumference $(\mathrm{cm})$ & $77.8 \pm 11.3$ & $77.8 \pm 12.1$ & 0.982 \\
\hline
\end{tabular}

Data are shown as mean \pm standard deviation or number (\%).

$\mathrm{BP}$, blood pressure. 


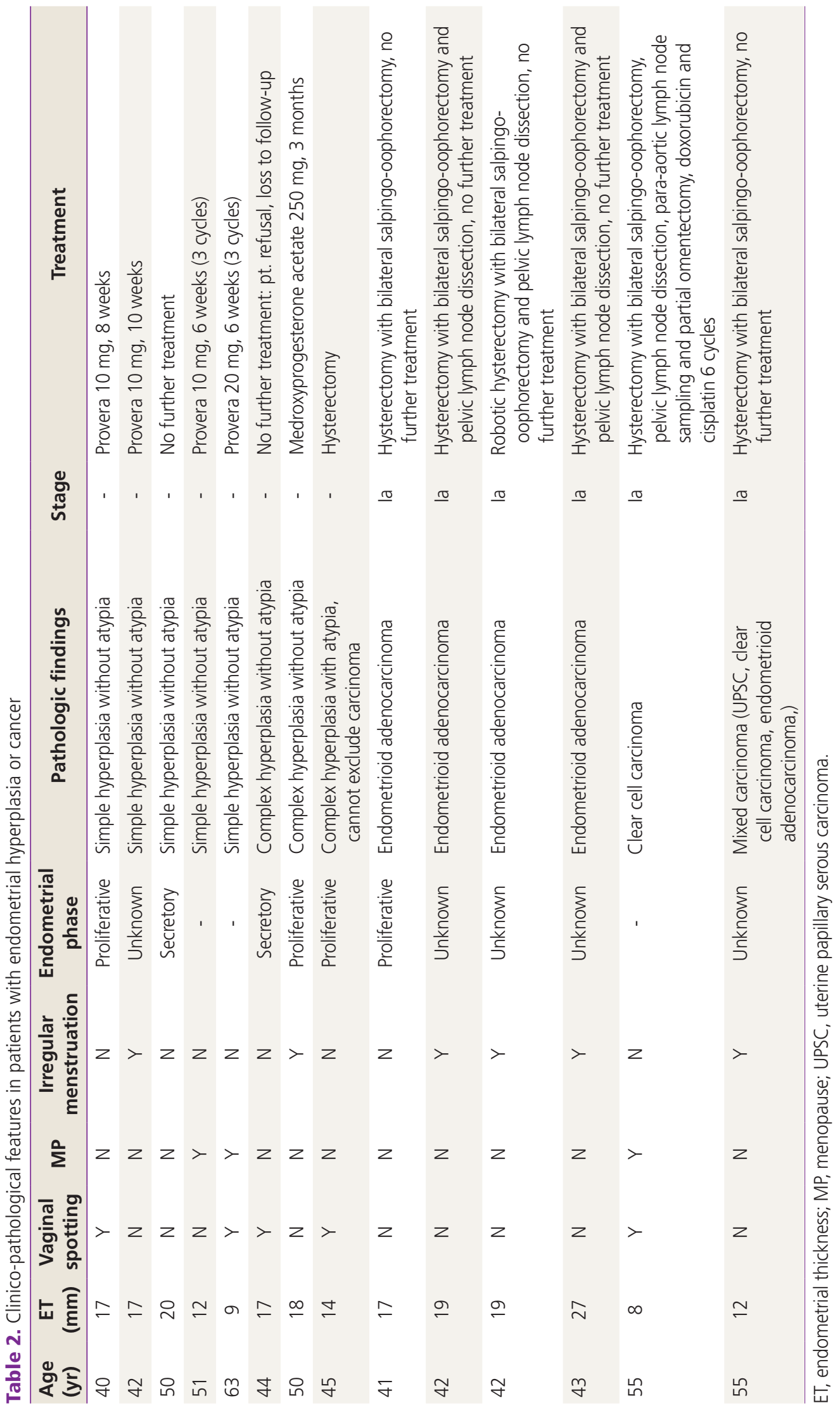




\section{Obstetrics \& Gynecology Science}

Vol. 62, No. 6, 2019

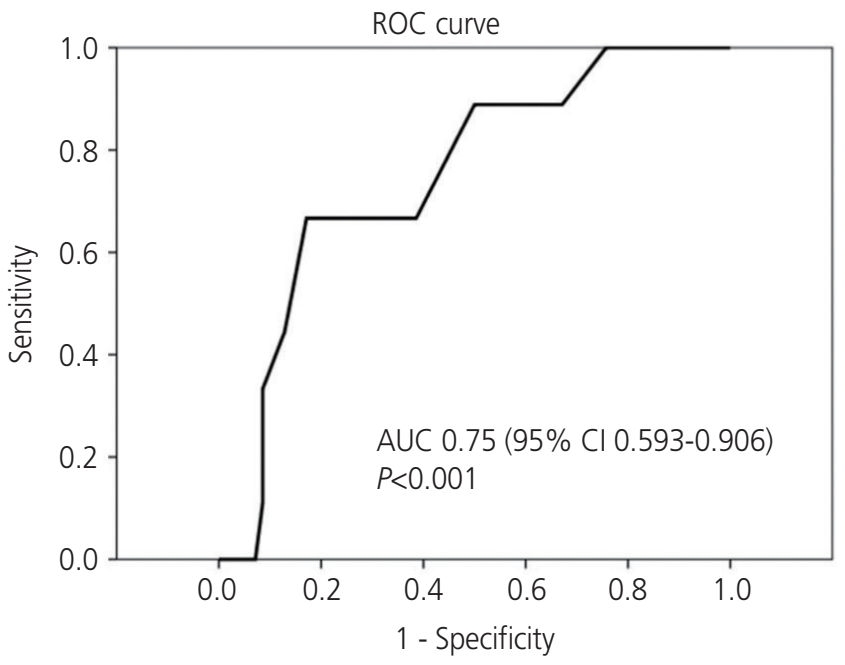

Fig. 2. Receiver operating characteristic (ROC) curve of endometrial thickness measurements for the detection of endometrial hyperplasia or cancer. AUC, area under the curve; $\mathrm{Cl}$, confidence interval.

women with an incidental finding of a thickened endometrium may be required to undergo endometrial assessment. The incidence of a thickened endometrium in asymptomatic postmenopausal women has been reported to range from $10 \%$ to $17 \%$ [16]. However, the optimal cut-off value for ET in these women has not been determined [17], and most women undergo invasive diagnostic evaluation, which may increase patient anxiety and medical costs.

This study enrolled women who visited the health screening and promotion center at Asan Medical Center. The patients enrolled were diagnosed with incidental endometrial abnormalities and did not have any need to visit a hospital despite the presence of mild symptoms (e.g., vaginal bleeding or spotting).

Endometrial pathology was initially defined as an ET of $\geq 5$ $\mathrm{mm}$ in postmenopausal women and $8 \mathrm{~mm}$ in the proliferative phase and $16 \mathrm{~mm}$ in the secretory phase in premenopausal women in this study. These values are clinically used criteria. However, there are no further guidelines for abnormal endometrial findings. All patients diagnosed with endometrial pathology on ultrasonography can be recommended to undergo invasive tests, such as endometrial biopsy (e.g., dilatation and curettage, hysteroscopic biopsy, or Pipelle endometrial sampling) or repetitive short-term ultrasonographic follow-up. However, this increases patients' anxiety for malignancy, increasing discomfort owing to invasive or repeated tests and total medical costs.

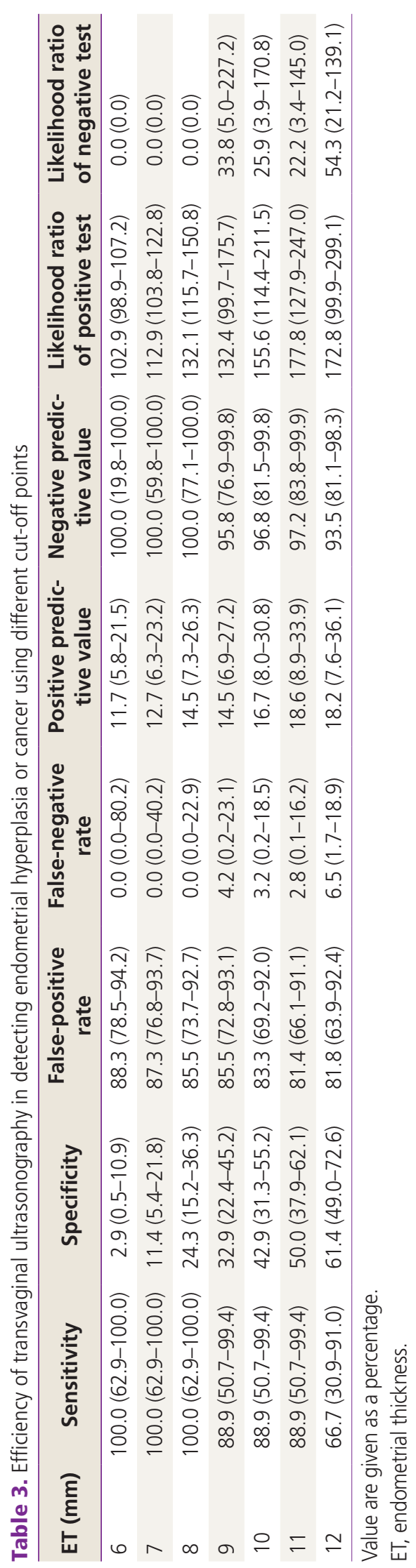




\section{Obstetrics \& Gynecology Science}

Yu Ran Park, et al. Endometrial thickness by sonography

This study investigated and showed the incidence of $\mathrm{EH}+$ or endometrial cancer among clinically asymptomatic women and supplementary data for patients with incidentally found endometrial abnormalities.

This study was conducted to investigate the endometrial pathology in patients who did not have any symptoms or clinically significant symptoms. Asymptomatic cases with endometrial polyp and submucosal myoma usually do not need any treatments because of their benign nature. We were interested in patients with premalignant or malignant lesions associated with endometrial pathology observed on ultrasonography. Most endometrial polyps and submucosal myomas are benign lesions in clinical settings. However, they can have varying sizes, morphologies, and numbers. We thought that including endometrial polyps and submucosal myomas might be a bias because their varying sizes could affect ET. Thus, we particularly considered them as exclusion criteria.

Our analysis showed that the sonographic measurement of ET was highly diagnostic in predicting endometrial pathology, with an optimal cut-off value of $8 \mathrm{~mm}$. This finding is meaningful because all diagnosed patients had early-stage endometrial cancer. The patients with $\mathrm{EH}+$ and even those with clear cell carcinoma and mixed cell carcinoma had a good prognosis. Because all endometrial carcinomas were of stage la, none required further treatment, except for the patient with clear cell carcinoma. In addition, none of these patients showed evidence of tumor recurrence, with remission persisting in all of them. Two of the patients in this study had type II endometrial cancer, which is associated with a poorer prognosis compared with type I endometrial cancer. Furthermore, the 5-year survival rates in patients with stage II and higher cancers are less than 50\% [18]. However, both of our patients with type II endometrial cancer had stage la tumors and a good prognosis. These findings indicate that TVS is useful diagnostically in the endometrial evaluation of preand postmenopausal women regardless of symptoms.

However, the cut-off value for ET in asymptomatic postmenopausal women remains unclear. A recent study in women with postmenopausal bleeding reported that an ET of $3 \mathrm{~mm}$ had a sensitivity of $97 \%$ and a specificity of $45.3 \%$; an ET of $4 \mathrm{~mm}$ had a sensitivity of $94.1 \%$ and a specificity of $66.8 \%$; and an ET of $5 \mathrm{~mm}$ had a sensitivity of $93.5 \%$ and a sensitivity of $74 \%$ for the detection of endometrial cancer [19]. Thus, TVS with a 3-mm cut-off value has a high sensitivity for detecting endometrial cancer and can identify women with postmenopausal bleeding who are highly unlikely to have endometrial cancer, thereby avoiding more invasive endometrial biopsy [19]. A prospective cohort observational study in 259 postmenopausal women assessed 3- and 5-mm cut-off values during TVS evaluation for endometrial carcinoma detection [20]. The mean ET was found to be $3.83 \pm 2.95 \mathrm{~mm}$ (range, $1-25 \mathrm{~mm}$ ); the sensitivity, specificity, positive predictive value, and negative predictive value were $62.2 \%, 93.9 \%, 68.3 \%$, and $92.2 \%$, respectively, for the 5-mm cut-off value [20]. However, previous studies conducted to determine the cut-off values for ET to exclude malignancy have reported equivocal results. The United Kingdom Collaborative Trial of Ovarian Cancer Screening, which included 37,038 asymptomatic women divided into risk groups according to a logistic regression model, found that a cut-off value for ET of $6.75 \mathrm{~mm}$ had a sensitivity of $84.3 \%$ and a specificity of $89.9 \%$ in the high-risk group [21].

The major strengths of the present study are the large sample size and the performance of all TVS examinations by 2 experienced gynecologists in a dedicated menopausal clinic, which maintained original data from patients over a 10-year period. All women had received a definitive histological diagnosis, which provided an optimal reference standard. These factors most likely increased the validity of our results.

The main limitations of this study were its retrospective nature and the very low incidence of pathology-related conditions in the cohort. Because insufficient information was available for very rare occurrences, this study included only 92 (33.9\%) of the 271 patients who underwent endometrial biopsy. We analyzed the cut-off value for ET without dividing the menopausal states and phases occurring in the endometrium. Many enrolled women forgot their LMP owing to irregular menstruation. For example, we reviewed 14 positive cases. The number of patients in the postmenopausal state was 3 . Among 11 premenopausal patients, there are 2 in the secretory phase and 4 in the proliferative phase. Five patients (45.5\%) forgot their LMP because of irregular menstruations. We thought that confirming the endometrial phase in perimenopausal women with irregular menstruation was difficult clinically. Thus, we calculated the cut-off value for ET without dividing the menopausal states and phases occurring in the endometrium.

We excluded pathologically proven endometrial polyps and submucosal myomas in this study. Thus, the application of the proposed cut-off value for endometrial pathologies 


\section{Obstetrics \& Gynecology Science}

Vol. 62, No. 6, 2019

that might be confused with endometrial polyps or submucosal myomas on ultrasonography is limited. However, the proposed cut-off value can be used to determine whether biopsy should be performed to confirm malignancy or a precancerous state in endometrial pathologies that can be distinguished from endometrial polyps or submucosal myomas on ultrasonography. Despite these limitations, many patients with similar demographic characteristics were included in the study, and good follow-up data were available. Moreover, the availability of 10-year data and definitive diagnosis most likely helped mitigate these limitations. Thus, we believe that the quality of our data was satisfactory.

In conclusion, TVS is useful for assessing endometrial pathology and diagnosing early endometrial cancer. Early detection of these conditions in patients with no specific symptoms (e.g., vaginal bleeding or spotting) can result in good patient outcomes. An ET of $\geq 8 \mathrm{~mm}$ was found to be the optimal cut-off value for detecting endometrial pathologies and cancers. Prospective studies with larger sample sizes are needed to confirm our findings.

\section{Conflict of Interest}

No potential conflict of interest relevant to this article was reported.

\section{Ethical approval}

This study was approved by the Institutional Review Board of Asan Medical Center (IRB No. 2016-0399), confirming its adherence to the Ethical Guidelines of the Declaration of Helsinki.

\section{Patient consent}

The requirement for obtaining informed consent was waived by the ethics review board owing to the retrospective nature of the study.

\section{References}

1. von Gruenigen VE, Gil KM, Frasure $H E$, Jenison EL, Hopkins MP. The impact of obesity and age on quality of life in gynecologic surgery. Am J Obstet Gynecol 2005;193:1369-75.

2. Bray F, Dos Santos Silva I, Moller H, Weiderpass E. Endometrial cancer incidence trends in Europe: underlying determinants and prospects for prevention. Cancer Epidemiol Biomarkers Prev 2005;14:1132-42.

3. Haslam DW, James WP. Obesity. Lancet 2005;366:1197209.

4. Malkasian GD Jr, Annegers JF, Fountain KS. Carcinoma of the endometrium: stage I. Am J Obstet Gynecol 1980;136:872-88.

5. Goldstein SR, Zeltser I, Horan CK, Snyder JR, Schwartz LB. Ultrasonography-based triage for perimenopausal patients with abnormal uterine bleeding. Am J Obstet Gynecol 1997;177:102-8.

6. Astrup K, Olivarius NF, Møller S, Gottschau A, Karlslund $W$. Menstrual bleeding patterns in pre- and perimenopausal women: a population-based prospective diary study. Acta Obstet Gynecol Scand 2004;83:197-202.

7. Hallberg L, Högdahl AM, Nilsson L, Rybo G. Menstrual blood loss--a population study. Variation at different ages and attempts to define normality. Acta Obstet Gynecol Scand 1966;45:320-51.

8. Fleischer AC, Wheeler JE, Lindsay I, Hendrix SL, Grabill S, Kravitz $B$, et al. An assessment of the value of ultrasonographic screening for endometrial disease in postmenopausal women without symptoms. Am J Obstet Gynecol 2001;184:70-5.

9. Karlsson B, Granberg S, Wikland M, Ylöstalo P, Torvid $K$, Marsal K, et al. Transvaginal ultrasonography of the endometrium in women with postmenopausal bleeding--a Nordic multicenter study. Am J Obstet Gynecol 1995;172:1488-94.

10. Gull B, Karlsson B, Milsom I, Granberg S. Can ultrasound replace dilation and curettage? A longitudinal evaluation of postmenopausal bleeding and transvaginal sonographic measurement of the endometrium as predictors of endometrial cancer. Am J Obstet Gynecol 2003;188:401-8.

11. Tsuda $H$, Nakamura $H$, Inoue $T$, Kawamura $N$, Adachi $K$, Bandera CA. Transvaginal ultrasonography of the endo- 


\section{Obstetrics \& Gynecology Science}

Yu Ran Park, et al. Endometrial thickness by sonography

metrium in postmenopausal Japanese women. Gynecol Obstet Invest 2005;60:218-23.

12. Timmermans A, Opmeer BC, Khan KS, Bachmann LM, Epstein E, Clark TJ, et al. Endometrial thickness measurement for detecting endometrial cancer in women with postmenopausal bleeding: a systematic review and meta-analysis. Obstet Gynecol 2010;116:160-7.

13. Saidi MH, Sadler RK, Theis VD, Akright BD, Farhart SA, Villanueva GR. Comparison of sonography, sonohysterography, and hysteroscopy for evaluation of abnormal uterine bleeding. J Ultrasound Med 1997;16:587-91.

14. Smith RA, Manassaram-Baptiste D, Brooks D, Doroshenk M, Fedewa S, Saslow D, et al. Cancer screening in the United States, 2015: a review of current American cancer society guidelines and current issues in cancer screening. CA Cancer J Clin 2015;65:30-54.

15. Lynch HT, Lynch JF. Lynch syndrome: history and current status. Dis Markers 2004;20:181-98.

16. Goldstein SR. Modern evaluation of the endometrium. Obstet Gynecol 2010;116:168-76.

17. van Hanegem N, Breijer MC, Khan KS, Clark TJ, Burger $\mathrm{MP}, \mathrm{Mol} \mathrm{BW}$, et al. Diagnostic evaluation of the endome- trium in postmenopausal bleeding: an evidence-based approach. Maturitas 2011;68:155-64.

18. Murphy KT, Rotmensch J, Yamada SD, Mundt AJ. Outcome and patterns of failure in pathologic stages I-IV clear-cell carcinoma of the endometrium: implications for adjuvant radiation therapy. Int J Radiat Oncol Biol Phys 2003;55:1272-6.

19. Wong AS, Lao TT, Cheung CW, Yeung SW, Fan HL, $\mathrm{Ng}$ PS, et al. Reappraisal of endometrial thickness for the detection of endometrial cancer in postmenopausal bleeding: a retrospective cohort study. BJOG 2016;123:439-46.

20. Kasraeian M, Asadi N, Ghaffarpasand F, Karimi AA. Value of transvaginal ultrasonography in endometrial evaluation of non-bleeding postmenopausal women. Climacteric 2011;14:126-31.

21. Jacobs I, Gentry-Maharaj A, Burnell M, Manchanda $R$, Singh $N$, Sharma $A$, et al. Sensitivity of transvaginal ultrasound screening for endometrial cancer in postmenopausal women: a case-control study within the UKCTOCS cohort. Lancet Oncol 2011;12:38-48. 